\title{
вмJ Global Health Preceding group A streptococcus skin and throat infections are individually associated with acute rheumatic fever: evidence from New Zealand
}

\author{
Jane Oliver, ${ }^{1}$ Julie Bennett (10 , ${ }^{2}$ Sally Thomas, ${ }^{2}$ Jane Zhang, ${ }^{2}$ Nevil Pierse, ${ }^{2}$ \\ Nicole J Moreland, ${ }^{3}$ Deborah A Williamson, ${ }^{1}$ Susan Jack, ${ }^{4}$ Michael Baker ${ }^{2}$
}

To cite: Oliver J, Bennett J, Thomas S, et al. Preceding group A streptococcus skin and throat infections are individually associated with acute rheumatic fever: evidence from New Zealand. BMJ Global Health 2021;6:e007038. doi:10.1136/ bmjgh-2021-007038

Handling editor Senjuti Saha

- Additional supplemental material is published online only. To view, please visit the journal online (http://dx.doi.org/10. 1136/bmjgh-2021-007038)

Received 29 July 2021

Accepted 17 November 2021

Check for updates

(c) Author(s) (or their employer(s)) 2021. Re-use permitted under CC BY-NC. No commercial re-use. See rights and permissions. Published by BMJ.

${ }^{1}$ The Peter Doherty Institute for Infection and Immunity, Department of Infectious Diseases, University of Melbourne, Melbourne, Victoria Australia

${ }^{2}$ Department of Public Health, University of Otago Wellington Wellington, New Zealand ${ }^{3}$ Department of Molecular Medicine and Pathology, University of Auckland, Auckland, New Zealand ${ }^{4}$ Southern District Health Board, Dunedin, Otago, New Zealand

Correspondence to Dr Julie Bennett; julie.bennett@otago.ac.nz

\section{ABSTRACT}

Introduction Acute rheumatic fever (ARF) is usually considered a consequence of group A streptococcus (GAS) pharyngitis, with GAS skin infections not considered a major trigger. The aim was to quantify the risk of ARF following a GAS-positive skin or throat swab.

Methods This retrospective analysis used pre-existing administrative data. Throat and skin swab data (1866981 swabs) from the Auckland region, New Zealand and antibiotic dispensing data were used (2010-2017). Incident ARF cases were identified using hospitalisation data (2010-2018). The risk ratio (RR) of ARF following swab collection was estimated across selected features and timeframes. Antibiotic dispensing data were linked to investigate whether this altered ARF risk following GAS detection.

Results ARF risk increased following GAS detection in a throat or skin swab. Māori and Pacific Peoples had the highest ARF risk 8-90 days following a GAS-positive throat or skin swab, compared with a GAS-negative swab. During this period, the RR for Māori and Pacific Peoples following a GAS-positive throat swab was $4.8(95 \% \mathrm{Cl}$ 3.6 to 6.4 ) and following a GAS-positive skin swab, the RR was $5.1(95 \% \mathrm{Cl} 1.8$ to 15.0$)$. Antibiotic dispensing was not associated with a reduction in ARF risk following GAS detection in a throat swab (antibiotics not dispensed (RR: 4.1, 95\% Cl 2.7 to 6.2), antibiotics dispensed (RR: $4.3,95 \% \mathrm{Cl} 2.5$ to 7.4 ) or in a skin swab (antibiotics not dispensed (RR: $3.5,95 \% \mathrm{Cl} 0.9$ to 13.9 ), antibiotics dispensed (RR: $2.0,95 \% \mathrm{Cl} 0.3$ to 12.1 ).

Conclusions A GAS-positive throat or skin swab is strongly associated with subsequent ARF, particularly for Māori and Pacific Peoples. This study provides the first population-level evidence that GAS skin infection can trigger ARF.

\section{INTRODUCTION}

Infection with group A streptococcus (GAS) can trigger an immune-mediated response in a small minority of people, resulting in acute rheumatic fever (ARF). ${ }^{1}$ The immunological processes by which GAS infections trigger ARF are poorly understood. ${ }^{2}$ For around $60 \%$ of ARF cases, permanent cardiac damage, termed rheumatic heart disease (RHD),

\section{Key questions}

What is already known?

- Acute rheumatic fever (ARF) has long been recognised as an immune-mediated complication of untreated group A streptococcus (GAS) pharyngitis.

- Emerging evidence, particularly from Australia and New Zealand, indicates that GAS skin infections may also be important in triggering ARF.

- Due to the rarity of ARF and absence of comprehensive linked data, no previous study has been large enough to quantify the ARF risk following a laboratory-confirmed GAS infection across a large cohort.

- Primary prevention of ARF often focuses on detection of the preceding GAS infection and delivering prompt antibiotic treatment before ARF occurs, with varying levels of effectiveness.

What are the new findings?

- ARF risk increased following GAS detection in a throat or skin swab. Māori and Pacific Peoples had the highest risk of developing ARF in the 8-90-day period following collection of a GAS positive throat or skin swab.

During this period, the risk ratio (RR) of ARF for Māori and Pacific Peoples following GAS-positive throat swab was 4.8 (95\% Cl 3.6 to 6.4), compared with GAS-negative throat swab.

- The RR of ARF for Māori and Pacific Peoples following GAS-positive skin swab during this period was $5.1(95 \% \mathrm{Cl} 1.8$ to 15.0$)$ compared with GASnegative skin swab.

- Dispensing antibiotics was not associated with a reduction in ARF risk following GAS detection in a throat or skin swab.

persists. ARF recurrences can worsen existing cardiac damage and produce new damage. Estimates indicate that in 2015, there were 34 million people living with RHD worldwide with 320000 associated deaths that year. ${ }^{3}$ ARF 


\section{Key questions}

What do the new findings imply?

- This study helps confirm GAS pharyngitis as a major trigger for ARF. It also provides the first large-scale evidence that GAS skin infection can also cause ARF

- The finding that antibiotic dispensing was not associated with a reduction in the risk of subsequent $A R F$ is extremely concerning.

- This result suggests that more effective interventions are needed to prevent progression from GAS infection to ARF.

- A new focus should be placed on addressing the burden of skin infections to reduce the risk of ARF in New Zealand.

produces an inequitable burden of disease, with the highest rates in low and middle-income countries and among some, often Indigenous-minority, populations living in high-income countries. In New Zealand, the rate of initial ARF hospitalisations for Indigenous Māori children aged 5-14 years was 36 per 100000 (2000-2018 inclusive), while the rate for Pacific children in this age group was 80 per 100000 population, representing some of the highest rates of ARF in the world. ${ }^{4}$

GAS pharyngitis is most common among 5-14-year-old children and causes around $37 \%$ of all pharyngitis episodes in this age group. ${ }^{5}$ Historically, ARF has been considered a consequence of untreated GAS pharyngitis. GAS pharyngitis is usually described in the literature as preceding ARF by around 2-3 weeks ${ }^{6}$ although ARF may take longer to present. One study of ARF ( $n=251$ cases) observed that $8 \%$ of cases occurred more than 45 days after GAS pharyngitis was diagnosed. ${ }^{7}$ GAS carriage is not thought to be associated with ARF. ${ }^{8}$

More recently, GAS skin infections have been proposed as triggering ARF, either directly or in combination with GAS pharyngitis. ${ }^{9}{ }^{10}$ New Zealand has a high and increasing incidence of skin infections, with the burden borne predominately by Māori and Pacific children. ${ }^{11-13}$

In New Zealand, rapid testing for GAS is not recommended, with GAS detected using laboratory swab culturing, which usually requires around 48 hours. Consequently, an empiric antibiotic prescription is recommend for people at high risk of ARF, where GAS pharyngitis is clinically suspected, with the patient instructed to cease taking antibiotics if the throat swab culture result turns out to be GAS negative. ${ }^{14}$ When diagnosing ARF, hospitalisation is standard-of-care for all suspected cases. A throat swab is routinely collected as part of the diagnostic workup. ${ }^{15}$ Around half of all national ARF cases reside in the Auckland region. ${ }^{4}$ An intensive ARF prevention programme has been operating there since 2012, which focuses on sore throat management. Here, throat swab culturing is used to detect GAS pharyngitis, with prompt antibiotic dispensing to treat the infection before ARF occurs, free of charge to eligible individuals. ${ }^{16}$

The high incidence of ARF in the Auckland region of New Zealand and the active sore throat management programme operating in that region, combined with highly integrated, routinely collected administrative health data, provides a unique opportunity to investigate the risk of ARF following a GAS-positive throat or skin swab. The aim of this study was to quantify the risk of ARF following a GAS-positive throat or skin swab by linking routinely collected administrative data.

\section{METHODS}

\section{Data sources and linkages}

In New Zealand, all publicly and privately funded hospital admissions are recorded in the national minimum data set (New Zealand Ministry of Health National Minimum Dataset; NMDS), which includes diagnostic information with international classification of diseases (ICD) coding. Labtests Ltd, the sole community pathology laboratory provider has serviced the whole Auckland region since late-2009, routinely collecting data on swabs it receives for processing, including the culture result. ${ }^{17}$ The National Pharmaceutical Collection contains claim and payment information from pharmacists for subsidised pharmaceutical dispensing, including antibiotic dispensing, with data on more than one billion scripts. ${ }^{18}$ The universal National Health Index (NHI) number enables the identification of individuals in health data and linkage of their information across data sets.

All ARF diagnoses corresponding to hospitalisations from 1988 to 2018 inclusive were obtained from the NMDS (ICD-10: I00-I02 and ICD-9: 390-392), as were all RHD diagnoses (ICD-10: I05-I09 and ICD-9: 393-398). The first admission with ARF/RHD as principal diagnosis for each patient was identified and all later entries excluded. All individuals with an admission for RHD preceding their first ARF hospitalisation were excluded. Admissions for non-New Zealand citizens were removed. Hospital transfers were excluded, so only the first record was included for each ARF hospitalisation. This first ARF hospitalisation was then taken to represent an initial episode of ARF. The data set was restricted to initial ARF hospitalisations from 2010 to 2018 inclusive. Data on patients' 2013 New Zealand Deprivation Index score (NZDep), prioritised ethnicity, age and sex were added to all data set entries using information contained in the NHI. The NZDep score is an ecological measure of socioeconomic deprivation based on national census data. ${ }^{19}$

Auckland is the largest city in New Zealand, with a population of approximately 1.4 million people at the 2013 census. At that time, the city had a prioritised ethnic population mix of 10\% Māori, 12\% Pacific Peoples, 21\% Asian and $57 \%$ European/Other. ${ }^{20}$ Prioritised ethnicity grouping allocates individuals to a single ethnic group based on a prioritised order of Māori, Pacific Peoples, Asian and European/Other. For example, if an individual identifies as being both Māori and European, that person will be classified as Māori for the purposes of data analysis.

We identified 23 antibiotic formulations likely to treat GAS infections using published literature relating to antimicrobial treatment of GAS (online supplemental 
appendix 1), guided by expert opinions from an experienced clinical microbiologist and a general practitioner. Dispensing data for these antibiotics were obtained from the National Pharmaceutical Collection through the Ministry of Health (2010-2017 inclusive). Data included the pharmaceutical type, dispensing date and the patient's NHI, through which dispensing data were linked to swab data. ${ }^{18}$ A patient was considered to have received antibiotics if dispensing occurred within 7 days either side of the swab collection date. This time window enabled occasions where antibiotics were prescribed empirically (ie, without waiting for a GAS-positive swab culture result) to be identified as well as occasions where there were delays in reporting the swab culture result.

\section{Microbiological testing}

Diagnostic throat and skin swab data with microbiological culture results (2010-2017 inclusive) and the patient's NHI were obtained from the sole diagnostic community laboratory service provider for the Auckland region, ${ }^{17}$ through a laboratory information system search for all panel codes related to throat, wound and skin swabs. Swabs were cultured onto tryptic soy sheep blood agar and incubated for 48 hours at $37^{\circ} \mathrm{C}$ in $5 \% \mathrm{CO}_{2}$. Plates were reviewed after 24 and 48 hours of incubation, and colonies indicating beta-haemolytic streptococci were identified. Prior to 2012, streptococcal grouping latex was used to identify beta-haemolytic streptococci. From 2012 onwards, MALDI-TOF MS Biotyper (Bruker, Germany) was used. Emm-typing for GAS strain identification is not used in routine diagnostic testing, so strain-typing data were unavailable. Antibiotic susceptibility testing was also not routinely performed. ${ }^{15}$ Clinical information, such as presenting symptoms or infection severity, was not available.

Staff at the Ministry of Health encrypted the NHI codes on all data sets. ARF hospitalisations were matched, using the encrypted NHI, with laboratory swab data to identify individuals who had had a throat or skin swab and were hospitalised with initial ARF during the study period. Individuals who had a throat or skin swab and an associated antibiotic dispensing event were also identified by matching the encrypted NHI across data sets.

\section{Statistical analyses}

Data analysis was performed using SAS V.9.4, GraphPad Prism V.9.0.0 and Microsoft Excel. Risk of initial ARF hospitalisations per 100000 swabs were calculated with $95 \%$ CIs by culture result. When investigating differences between reported proportions, the $\chi^{2}$ test was used. Findings were considered statistically significant if $\mathrm{p}<0.05$. The risk ratio (RR) of initial ARF hospitalisation following a GAS-positive throat or skin swab was calculated across specified time periods, demographic and microbiological features. The comparison group was initial ARF hospitalisations for patients with an associated swab that was negative for GAS and group C/G streptococci ('GAS negative'). Where both a GAS-positive throat swab and a GAS-positive skin swab (14-days either side of one another) preceded initial ARF during the period of interest, these cases were identified and excluded from further analysis.

Our analyses focus on GAS pharyngitis or skin infection where swab collection preceded the initial ARF hospitalisation by $8-90$ days for children in the highest risk age group. Restricting analyses to this time period and age group maximised the sensitivity for detecting potentially causative infections.

\section{RESULTS}

\section{Prevalence of GAS positive throat and skin swabs}

We included all throat (1 430 183) and skin (436 798) swab culture results for the Auckland population over an 8-year period (2010-2017). GAS was detected in $14.3 \%$ of throat swabs and $12.7 \%$ of skin swabs. Māori and Pacific Peoples had a slightly higher proportion of GAS detection in throat swabs compared with European/Others (Māori RR: 1.03 Pacific RR: 1.01, $\mathrm{p}<0.01$, figure 1A). Māori and Pacific children had a markedly higher prevalence of GAS detection in skin swabs compared with European/Others (Māori RR: 4.93, Pacific RR: 5.37 p<0.0001; figure 1B).

\section{ARF following concurrent GAS-positive skin and throat swabs} Seven people had concurrent $( \pm 14$ days) GAS-positive skin and throat swabs 8-90 days prior to hospitalisation for ARF (3.4 per 100000 GAS-positive swabs). The risk of initial ARF hospitalisation was increased following concurrent GAS-positive throat and skin swabs compared with when throat and skin swabs were GAS negative (RR: 17.1, 95\% CI 4.5 to 104.0). These seven cases were excluded from subsequent analyses.

\section{Risk of ARF following GAS-positive swabs}

The ARF risk was highest for Māori and Pacific children aged 10-19 years and was concentrated during the 8-90 days following swab collection (figure 2A,B). For every 100000 GAS-positive throat swabs, 315 Māori/ Pacific children aged 10-19 years were hospitalised with initial ARF within 365 days of throat swab collection (figure 2A). For every 100000 GAS-positive skin swabs, 151 Māori/Pacific children aged 10-19 years were hospitalised with a diagnosis of initial ARF within 365 days of skin swab collection (figure 2B).

\section{ARF following a GAS-positive throat swab}

The risk of initial ARF hospitalisation was markedly increased in the 8-90 days following collection of a GASpositive throat swab, RR: $5.1,95 \%$ CI 3.8 to 6.9 (table 1). Children aged 10-14 years had a particularly high risk of initial ARF hospitalisations 8-90 days following a GASpositive throat swab (105.6 per 100000 throat swabs). The risk appeared to increase with age group; however, there were small ARF case numbers among those aged older than 19 years.

Females had a higher risk of ARF in the 8-90 days following a GAS-positive throat swab than males (RR: 

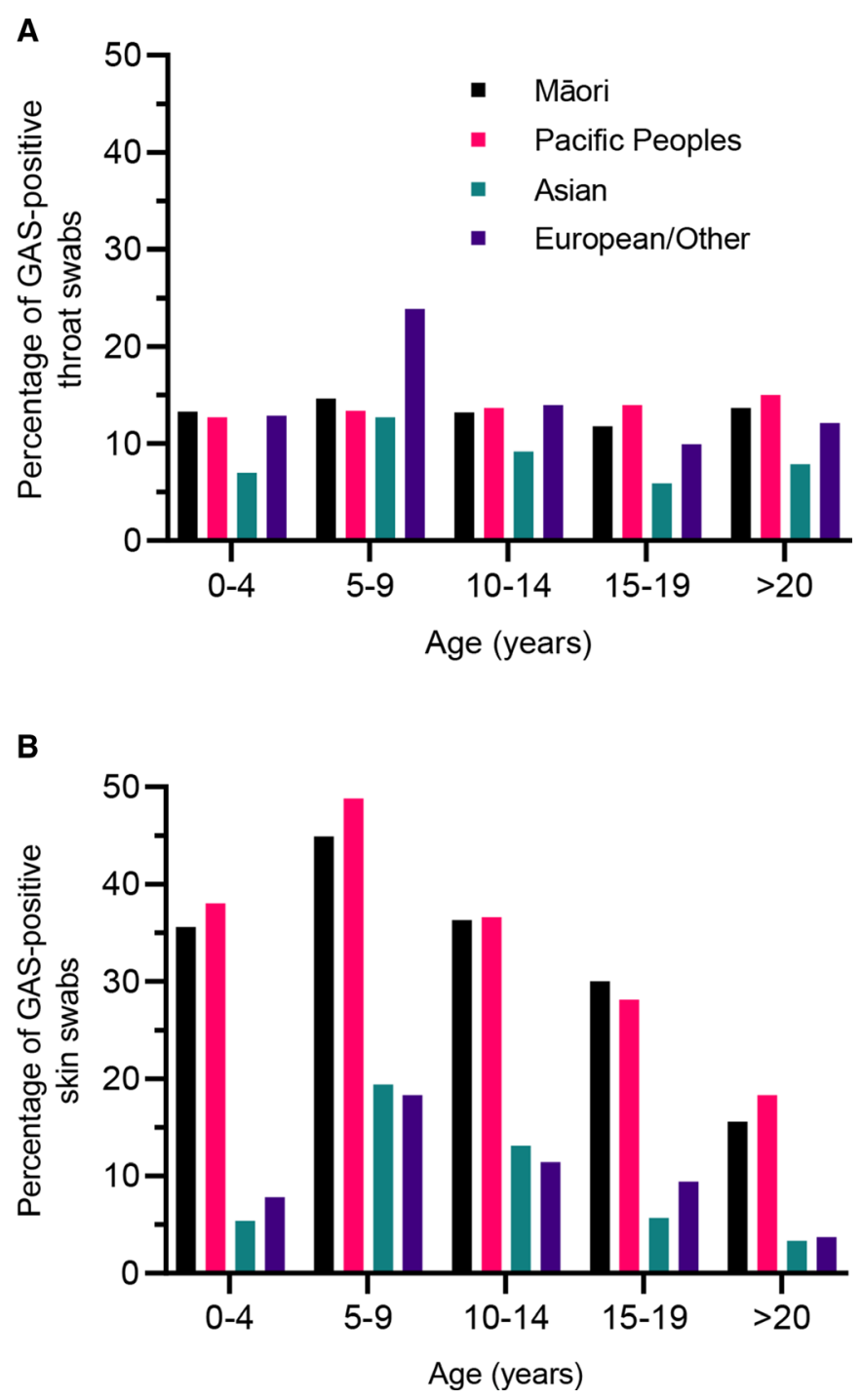

Figure 1 Prevalence (\%) of group A streptococcus (GAS) detection in throat swabs (A) and skin swabs (B) by age group and prioritised ethnicity, Auckland, 2010-2017.

5.6, 95\% CI 3.8 to 8.6). Māori and Pacific Peoples had an increased risk of ARF during this period (Māori RR: 5.8, 95\% CI 3.3 to 10.3 , Pacific RR: 4.5 , 95\% CI 3.7 to 6.3 ). Low case numbers meant that risk could not be assessed for other ethnicities (table 1).

When antibiotics were dispensed within 7 days of a GAS-positive throat swab collection, there was no statistically significant reduction in the risk of initial ARF hospitalisation 8-90 days following swab collection: Antibiotics were not dispensed (RR: 4.1, 95\% CI 2.7 to 6.2 ), antibiotics dispensed (RR: 4.3, 95\% CI 2.5 to 7.4; table 1).

\section{ARF following GAS-positive skin swab}

The risk of initial ARF hospitalisation was highest, 8-90 days following a GAS-positive skin swab (RR: 15.5, 95\% CI 5.4 to 44.5 ) (table 2). Children aged 10-14 years had a particularly high risk of initial ARF hospitalisation 8-90 days following a GAS-positive swab (94.7 per 100000 GAS-positive skin swabs). Males had a higher risk of initial ARF during this period (RR: $21.3,95 \%$ CI 4.4 to 102.6 ), as
A

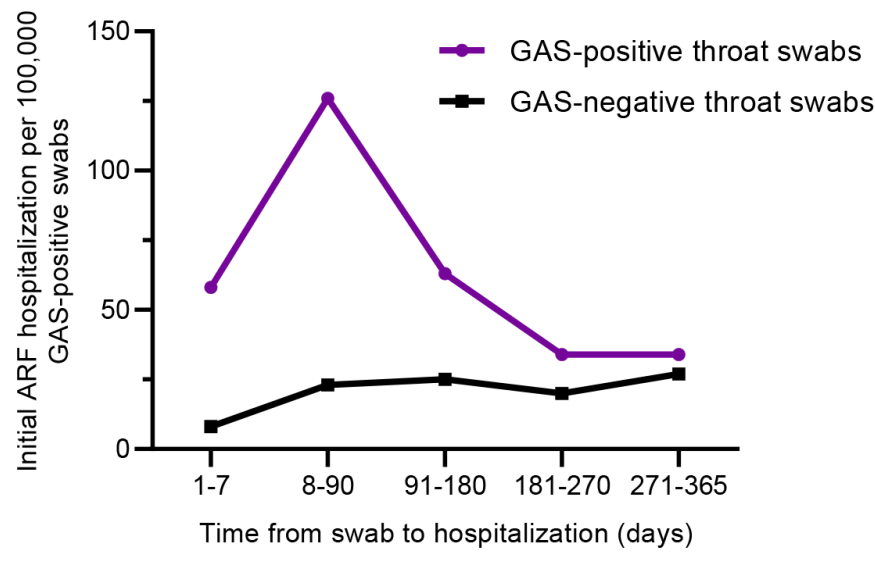

B

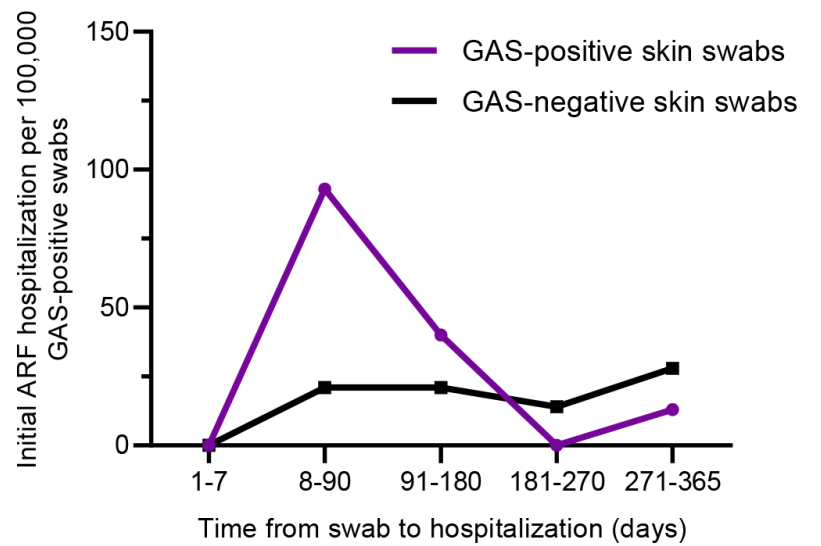

Figure 2 Risk of acute rheumatic fever (ARF) hospitalisation (2010-2018) following a group A streptococcus (GAS) positive throat swab (A) or skin swab (B) compared with group A streptococcus negative swabs, by time-period after swab collection (2010-2017), Māori and Pacific children aged 10-19 years.

did people living in areas of high socioeconomic deprivation (Quintiles 4/5). Māori and Pacific Peoples had an increased risk of initial ARF 8-90 days following GASpositive swab collection, with findings for Pacific People most apparent in the 10-19year age group (RR: 6.1, $95 \%$ CI 1.2 to 30.4 ). Low case numbers meant that risk could not be assessed for other ethnicities (table 2).

When antibiotics were dispensed within 7 days of GASpositive skin swab collection, no statistically significant reduction in the risk of initial ARF hospitalisation was noted over the subsequent 8-90-day period: antibiotics not dispensed (RR: 3.5 , 95\% CI 0.9 to 13.9 ), antibiotics dispensed (RR: 2.0, 95\% CI 0.3 to 12.1).

\section{DISCUSSION}

This study provides a clear link from GAS detection in throat and skin swabs to the subsequent development of ARF in a large population, observed over an extended period of time (2010-2018). Results indicate that both GAS pharyngitis and GAS skin infections are strongly associated with the development of ARF. There was a 
Table 1 Risk of initial acute rheumatic fever hospitalisation (2010-2018) following a GAS positive throat swab (2010-2017), Auckland, New Zealand

\begin{tabular}{|c|c|c|c|c|c|c|}
\hline \multicolumn{3}{|c|}{ ARF risk following GAS positive throat swab* } & \multicolumn{3}{|c|}{ ARF risk following GAS negative throat swabł* } & $\begin{array}{l}\text { RR } \\
\text { Risk of ARF following } \\
\text { GAS-positive swab }\end{array}$ \\
\hline $\begin{array}{l}\text { Total ARF } \\
\text { following GAS } \\
\text { positive swab }\end{array}$ & $\begin{array}{l}\text { Total GAS } \\
\text { positive } \\
\text { swabs }\end{array}$ & $\begin{array}{l}\text { ARF per } \\
100000 \text { GAS } \\
\text { positive swabs }\end{array}$ & $\begin{array}{l}\text { Total ARF } \\
\text { following GAS } \\
\text { negative swab }\end{array}$ & $\begin{array}{l}\text { Total GAS } \\
\text { negative } \\
\text { swabs }\end{array}$ & $\begin{array}{l}\text { ARF per } \\
100000 \text { GAS } \\
\text { negative } \\
\text { swabs }\end{array}$ & $\begin{array}{l}\text { Risk of ARF following } \\
\text { GAS-positive swab } \\
\text { versus GAS-negative } \\
\text { swab } \\
\text { RR }(95 \% \mathrm{Cl})\end{array}$ \\
\hline
\end{tabular}

Risk of initial ARF hospitalisation during specific time window (total population)

\begin{tabular}{lrrrrrrr} 
ARF 1-7 days of swab & 34 & 204085 & 16.7 & 35 & 1223456 & 2.9 & 5.82 (3.63 to 9.34) \\
\hline ARF 8-90 days of swab & 84 & 204085 & 41.2 & 99 & 1223456 & 8.0 & 5.14 (3.84 to 6.88) \\
\hline ARF 1-365 days of swab & 236 & 204084 & 115.6 & 599 & 1223456 & 49.0 & 2.36 (2.03 to 2.75)
\end{tabular}

Risk of initial ARF hospitalisation, restricted to 8-90 days

Age group (years) (8-90 days following swab)

\begin{tabular}{|c|c|c|c|c|c|c|c|}
\hline$<5$ & 0 & 14954 & - & 0 & 108358 & - & - \\
\hline $5-9$ & 24 & 95942 & 25.0 & 40 & 502493 & 8.0 & 3.14 (1.89 to 5.21$)$ \\
\hline $10-14$ & 49 & 46380 & 105.6 & 51 & 281904 & 18.1 & 5.64 (3.93 to 8.67$)$ \\
\hline $15-19$ & 7 & 11539 & 60.7 & 5 & 86256 & 5.8 & 10.47 (3.32 to 32.97$)$ \\
\hline $20-29$ & 4 & 13133 & 30.5 & 2 & 83588 & 2.4 & 12.73 (2.33 to 69.49$)$ \\
\hline$>29$ & 0 & 22137 & - & 0 & 160857 & - & - \\
\hline \multicolumn{8}{|c|}{ Gender (8-90 days following a swab) } \\
\hline Male & 40 & 98433 & 40.6 & 48 & 546398 & 8.8 & $4.63(3.04$ to 7.04$)$ \\
\hline Female & 44 & 105652 & 41.6 & 50 & 677058 & 7.4 & $5.64(3.76$ to 8.64$)$ \\
\hline \multicolumn{8}{|c|}{ Prioritised ethnicity (8-90 days following a swab) } \\
\hline Māori & 23 & 44990 & 50.0 & 23 & 266521 & 8.6 & $5.79(3.25$ to 10.33$)$ \\
\hline Pacific & 57 & 80854 & 70.5 & 73 & 476335 & 15.7 & 4.48 (3.70 to 6.32$)$ \\
\hline Māori and Pacific & 80 & 126844 & 63.1 & 98 & 742856 & 13.2 & 4.78 (3.56 to 6.42$)$ \\
\hline Asian & 0 & 14221 & - & 0 & 132879 & - & - \\
\hline NZ European/Other & 4 & 63020 & 6.3 & 0 & 347721 & - & - \\
\hline \multicolumn{8}{|c|}{ Socio-economic deprivation (NZ Dep quintile) (8-90 days following a swab) } \\
\hline 1 (low deprivation) & 1 & 23842 & 4.2 & 0 & 132310 & - & - \\
\hline 2 & 3 & 23693 & 12.7 & 5 & 134763 & 3.7 & $3.41(0.82$ to 14.28$)$ \\
\hline 3 & 6 & 19834 & 30.3 & 3 & 108675 & 2.8 & $10.96(2.74$ to 43.83$)$ \\
\hline 4 & 12 & 22591 & 53.1 & 15 & 131431 & 11.4 & 4.65 (2.18 to 9.94$)$ \\
\hline 5 (high deprivation) & 62 & 114125 & 54.3 & 75 & 616277 & 12.2 & 5.19 (3.71 to 7.26$)$ \\
\hline \multicolumn{8}{|c|}{ Risk of initial ARF hospitalisation, during 8-90 day window restricted to Māori and Pacific aged 10-19 years } \\
\hline \multicolumn{8}{|c|}{ Gender (8-90 days following a swab) } \\
\hline Male & 25 & 20673 & 120.9 & 28 & 113699 & 24.6 & 4.91 (2.86 to 8.42$)$ \\
\hline Female & 27 & 20496 & 131.7 & 28 & 129348 & 21.6 & 6.09 (3.59 to 10.32$)$ \\
\hline \multicolumn{8}{|c|}{ Prioritised ethnicity (8-90 days following a swab) } \\
\hline Māori & 14 & 13737 & 101.9 & 18 & 85588 & 21.0 & 4.85 (2.41 to 9.74$)$ \\
\hline Pacific & 38 & 27432 & 138.5 & 38 & 157457 & 24.1 & 5.74 (3.64 to 8.99$)$ \\
\hline \multicolumn{8}{|c|}{ Socio-economic deprivation (NZ Dep quintile) (8-90 days following a swab) } \\
\hline 1 (low deprivation) & 0 & 680 & - & 0 & 3987 & - & - \\
\hline 2 & 2 & 1575 & 127.0 & 5 & 8882 & 56.3 & 2.26 (0.22 to 11.62$)$ \\
\hline 3 & 4 & 1910 & 209.4 & 3 & 9794 & 30.6 & 6.84 (1.53 to 30.52 ) \\
\hline 4 & 5 & 4237 & 118.0 & 8 & 23053 & 34.7 & 3.40 (1.11 to 10.39 ) \\
\hline 5 (high deprivation) & 41 & 32767 & 125.1 & 40 & 197330 & 20.3 & 6.17 (3.99 to 9.54$)$ \\
\hline Total & 52 & 41169 & 126.3 & 55 & 243047 & 23.0 & 5.48 (3.76 to 7.99$)$ \\
\hline \multicolumn{8}{|c|}{ Antibiotics dispensed (within 7 days of swab among Māori and Pacific People aged 5-19 years) } \\
\hline Antibiotics & 43 & 52730 & 81.5 & 19 & 100107 & 19.0 & $4.30(2.50$ to 7.37$)$ \\
\hline No antibiotics & 33 & 55744 & 59.2 & 77 & 534334 & 14.4 & $4.11(2.73$ to 6.18$)$ \\
\hline
\end{tabular}

Boldface values relate to the Total data. Significance is $P>0.05$.

*GAS-positive skin swabs were excluded \pm 14 days of all throat swabs.

TGAS-negative swabs were also culture negative for group C/G streptococci.

ARF, acute rheumatic fever; GAS, group A streptococcus; RR, risk ratio. 
Table 2 Risk of initial ARF hospitalisation (2010-2018) following a GAS positive skin swab (2010-2017), Auckland, New Zealand

\begin{tabular}{|c|c|c|c|c|c|c|}
\hline \multicolumn{3}{|c|}{ ARF risk following GAS positive skin swab* } & \multicolumn{3}{|c|}{ ARF risk following GAS negative skin swabł* } & $\mathbf{R R}$ \\
\hline $\begin{array}{l}\text { Total ARF } \\
\text { following GAS } \\
\text { positive swab }\end{array}$ & $\begin{array}{l}\text { Total GAS } \\
\text { positive } \\
\text { swabs }\end{array}$ & $\begin{array}{l}\text { ARF per } \\
100000 \text { GAS } \\
\text { positive } \\
\text { swabs }\end{array}$ & $\begin{array}{l}\text { Total ARF } \\
\text { following GAS } \\
\text { negative swab }\end{array}$ & $\begin{array}{l}\text { Total GAS } \\
\text { negative } \\
\text { swabs }\end{array}$ & $\begin{array}{l}\text { ARF per } \\
100000 \text { GAS } \\
\text { negative swabs }\end{array}$ & $\begin{array}{l}\text { Risk of ARF following } \\
\text { GAS-positive swab vs } \\
\text { GAS-negative swab } \\
\text { RR(95\% Cl) }\end{array}$ \\
\hline
\end{tabular}

Risk of initial ARF hospitalisation during specific time window (total population)

\begin{tabular}{lrrrrrrr} 
ARF 1-7 days of swab & 2 & 54217 & 3.7 & 0 & 380755 & - & - \\
ARF 8-90 days of swab & 11 & 54217 & 20.3 & 5 & 380755 & 1.3 & 15.45 (5.37 to 44.48) \\
\hline ARF 1-365 days of swab & 24 & 54217 & 44.3 & 27 & 380755 & 7.5 & 6.24 (3.60 to 10.82)
\end{tabular}

Risk of initial ARF hospitalisation, restricted to 8-90 days

Age group (years) (8-90 days following swab)

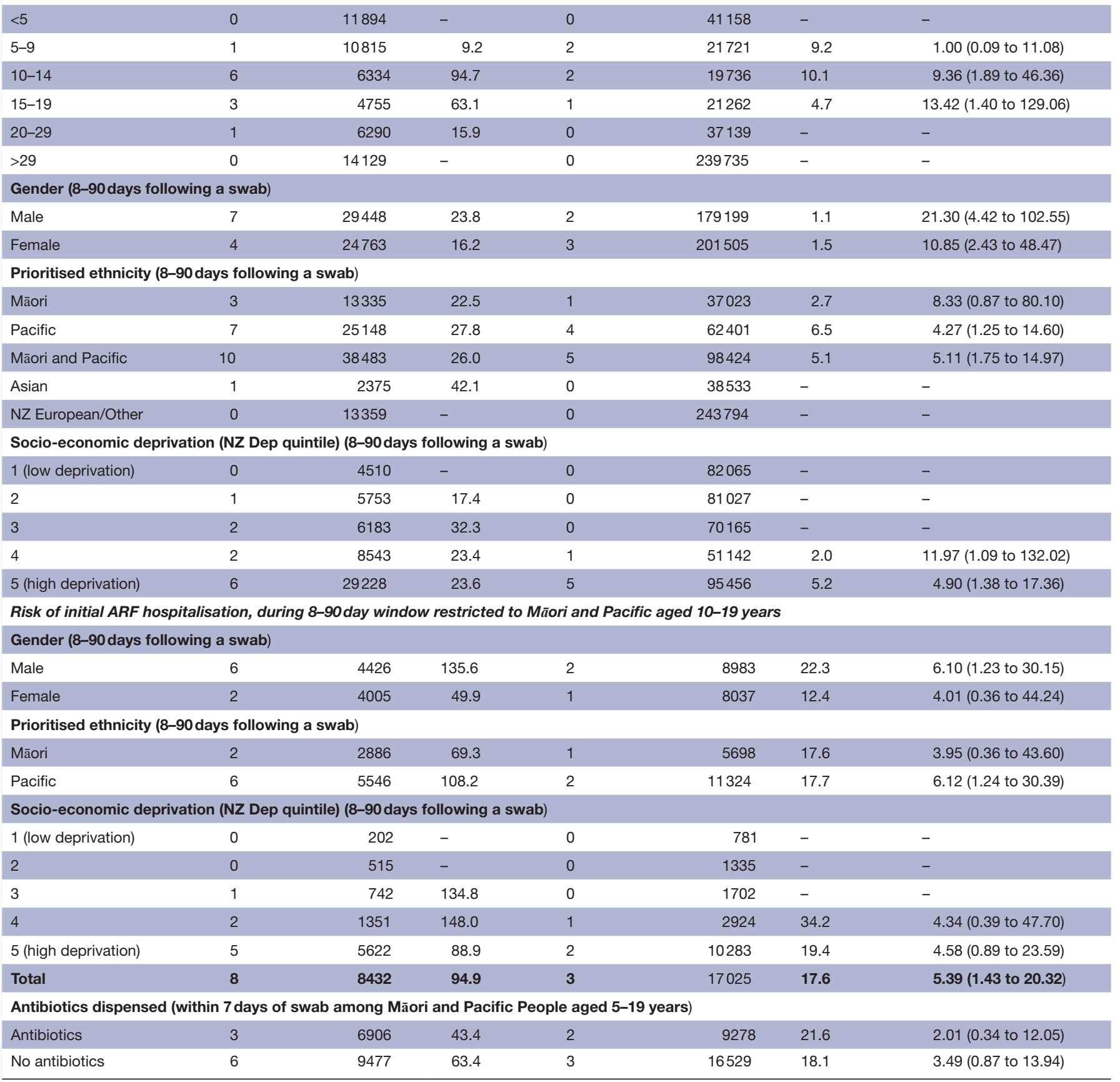

${ }^{*}$ GAS-positive throat swabs were excluded \pm 14 days of all skin swabs.

tGAS-negative swabs were also culture negative for group C/G streptococci.

ARF, acute rheumatic fever; GAS, group A streptococcus; RR, risk ratio. 
significant increase in the ARF risk for Māori and Pacific Peoples during the 8-90-day latency period following GAS detection from a throat or skin swab. This risk was markedly higher than the baseline risk for Māori and Pacific Peoples who had a skin or throat swab collected, but GAS was not detected. These findings support the well-established clinical view that GAS pharyngitis is an important initiator of ARF. ${ }^{21}$ They also add new evidence that GAS skin infection has a similar propensity to cause ARF, both alone and in combination with concurrent pharyngitis. Dispensing antibiotics was not associated with a reduction in ARF risk following detection of GAS in a throat or skin swab. This finding is extremely concerning and implies that ARF primary prevention interventions may be failing to protect high-risk groups.

While a causal association between the presence of an organism and the occurrence of disease cannot be confirmed using observational data alone, this analysis comprehensively evaluates the risk of an initial ARF hospitalisation by swab culture result in a large population. The ARF risk is likely underestimated by these analyses as cases of GAS pharyngitis may not come to clinical attention and GAS skin infection may be treated empirically within primary care without a swab. The absence of a protective effect following antibiotic dispensing may be due to poor compliance with treatment or may indicate the importance of immune priming following repeated GAS infections. ${ }^{22}$ A major strength of this study is that the exposed population (ie, GAS culture positive individuals) are being compared with a demographically very similar but unexposed population (ie, GAS culture negative individuals), thus controlling for major confounders. Furthermore, the data sources are likely fairly complete, given that hospitalisation is recommended as standard of care for all suspected ARF cases in New Zealand, and rapid testing for GAS is not recommended. ${ }^{14}{ }^{15}$ However, it is possible that there are systematic differences between GAS-positive and negative groups. For example, GAS detection could be a consequence of household crowding, which in turn may increase the risk of ARF through repeated environmental exposures to GAS. ${ }^{23}$ As case numbers were low, we were not able to assess whether the risk of ARF increased with repeated GAS detection.

Māori and Pacific children have among the highest rates of ARF in the world and experience health disparities across many chronic and acute conditions, and inequitable socioeconomic deprivation. ${ }^{24}{ }^{25}$ While the pathogenesis of ARF is not fully elucidated, a clearly increased risk was observed for Māori and Pacific children following GAS detection in a throat or skin swab. $\mathrm{ARF}$ is triggered following a complex interplay of susceptibility, immunological and environmental factors. ${ }^{23} 26$ Immune priming from repeated GAS exposures likely plays a major pathogenic role, especially given that no genetic risk factors have been consistently identified. ${ }^{23} 24$ A further indication of immune priming in early childhood is demonstrated in our study by the higher GAS detection in throat swabs for European/other children aged 5-9 years, but no corresponding ARF risk. ARF is still a rare disease in New Zealand. Although ARF is usually thought to follow GAS infection by around 3 weeks, ${ }^{6}$ low case numbers necessitated the use of the 8-90-day period to maximise sensitivity for detecting causative infections.

As pre-existing administrative data were used for these analyses, the data may be affected by errors, including misdiagnosis and miscoding. The sensitivity of ICD coding for ARF would have changed during the study period as the diagnostic criteria were refined and awareness of ARF increased with the recent national prevention programme. Despite this, the NMDS was estimated to be $79 \%$ sensitive for detecting true cases of ARF during 2011-2015. ${ }^{27}$ Swab data are likely highly complete as these are provided by the sole community service provider for the Auckland region. ${ }^{17}$ These data are also likely to reflect cases where throat or skin infection was clinically suspected. It is not normal practice to use swab culturing to screen for GAS carriage, nor is treatment of carriage recommended. ${ }^{14}$ While antibiotic dispensing would be captured in the National Pharmaceutical Collection, ${ }^{16}$ topical antiseptics purchased without prescription are not, so dispensing data may correspond to more severe skin infections. Regardless, no protective effect from antibiotic dispensing was observed on the risk of developing ARF 8-90 days after GAS detection in either a throat or a skin swab.

\section{CONCLUSION}

Prompt antibiotic treatment of GAS infections in groups with a known high risk of $\mathrm{ARF}$ is required to terminate the infective process, reducing the risk of poor outcomes, and to limit GAS transmission. Targeted sore throat management interventions should remain a key strategy in the prevention of ARF. A new focus should also be placed on addressing the burden of skin infections to reduce the risk of ARF in New Zealand. Such an approach would mirror strategies that led to dramatic reduction in ARF in Cuba and Costa Rica. ${ }^{28}$ Multifactorial interventions should aim to reduce socioeconomic deprivation, improve housing conditions, lower household crowding, improve access to healthcare and raise health literacy. ${ }^{23}$ These measures can supplement primary prevention interventions targeting GAS infections, aiming for a meaningful and sustained reduction in ARF in New Zealand.

Acknowledgements We would like to thank the staff at the community laboratory for providing the throat and skin swab data; in particular, we thank Dr Arlo Upton and Susan Smith.

Contributors J0 designed the analysis and interpreted the results, ST designed the skin infection analysis and interpreted the results, JB wrote the manuscript, interpreted the results and is the guarantor, JZ conducted the data analysis, NP provided biostatical support and supervision, NJM interpreted data, DAW interpreted data, SJ interpreted the data, MB conceived the project and supervised it. All authors have read and reviewed the manuscript.

Funding The work was supported by the Health Research Council of New Zealand, award number 16/005. J0 was funded by the Lotteries Health Research with the provision of a full-time $\mathrm{PhD}$ scholarship. ST received a training stipend from the New Zealand College of Public Health Medicine during her Masters of 
Public Health. DAW is supported by National Health and Medical Research Council Australia (NHMRC) Investigator Grant (APP1174555).

Competing interests None declared.

Patient consent for publication Not applicable.

Ethics approval Ethics approval for this study was obtained from the University of Otago, Wellington, New Zealand (Human Ethics Committee reference HD14/12) and the Northern B Health and Disability Ethics Committee, New Zealand (reference 14/ NTB/16).

Provenance and peer review Not commissioned; externally peer reviewed.

Data availability statement Data may be obtained from a third party and are not publicly available. Not applicable.

Supplemental material This content has been supplied by the author(s). It has not been vetted by BMJ Publishing Group Limited (BMJ) and may not have been peer-reviewed. Any opinions or recommendations discussed are solely those of the author(s) and are not endorsed by BMJ. BMJ disclaims all liability and responsibility arising from any reliance placed on the content. Where the content includes any translated material, BMJ does not warrant the accuracy and reliability of the translations (including but not limited to local regulations, clinical guidelines, terminology, drug names and drug dosages), and is not responsible for any error and/or omissions arising from translation and adaptation or otherwise.

Open access This is an open access article distributed in accordance with the Creative Commons Attribution Non Commercial (CC BY-NC 4.0) license, which permits others to distribute, remix, adapt, build upon this work non-commercially, and license their derivative works on different terms, provided the original work is properly cited, appropriate credit is given, any changes made indicated, and the use is non-commercial. See: http://creativecommons.org/licenses/by-nc/4.0/.

ORCID iD

Julie Bennett http://orcid.org/0000-0002-3461-7223

\section{REFERENCES}

1 Cunningham MW. Streptococcus and rheumatic fever. Curr Opin Rheumatol 2012;24:408-16.

2 Karthikeyan G, Guilherme L. Acute rheumatic fever. Lancet 2018;392:161-74.

3 Watkins DA, Johnson CO, Colquhoun SM, et al. Global, regional, and national burden of rheumatic heart disease, 1990-2015. N Engl J Med 2017;377:713-22.

4 Bennett J, Zhang J, Leung W, et al. Rising ethnic inequalities in acute rheumatic fever and rheumatic heart disease, New Zealand, 2000-2018. Emerg Infect Dis 2021;27:36-46.

5 Shaikh N, Leonard E, Martin JM. Prevalence of streptococcal pharyngitis and streptococcal carriage in children: a meta-analysis. Pediatrics 2010;126:e557-64.

6 Sika-Paotonu D, Beaton A, Raghu A, et al. Acute Rheumatic Fever and Rheumatic Heart Disease. In: Ferretti JJ, Stevens DL, Fischetti VA, eds. Streptococcus pyogenes : Basic Biology to Clinical Manifestations. Oklahoma City (OK), 2016.

7 Rammelkamp CH, Stolzer BL. The latent period before the onset of acute rheumatic fever. Yale J Biol Med 1961;34:386.

8 Shulman ST, Bisno AL, Clegg HW, et al. Clinical practice guideline for the diagnosis and management of group A streptococcal pharyngitis: 2012 update by the infectious diseases Society of America. Clin Infect Dis 2012;55:1279-82.

9 McDonald M, Currie BJ, Carapetis JR. Acute rheumatic fever: a chink in the chain that links the heart to the throat? Lancet Infect Dis 2004;4:240-5.

10 O'Sullivan L, Moreland NJ, Webb RH, et al. Acute rheumatic fever after group A Streptococcus pyoderma and group G Streptococcus pharyngitis. Pediatr Infect Dis J 2017;36:692-4.

11 O'Sullivan CE, Baker MG, Zhang J. Increasing hospitalizations for serious skin infections in New Zealand children, 1990-2007. Epidemiol Infect 2011;139:1794-804.

12 Berry S, Morton S, Atatoa Carr P, et al. Colonisation with Staphylococcus aureus and Streptococcus pyogenes in New Zealand preschool children. N Z Med J 2015;128:60-7.

13 Thomas S, Bennett J, Jack S, et al. Descriptive analysis of group A Streptococcus in skin swabs and acute rheumatic fever, Auckland, New Zealand, 2010-2016. Lancet Reg Health West Pac 2021;8:100101.

14 Heart Foundation of New Zealand. Group A streptococcal sore throat management guideline. 2014 update. Auckland: Heart Foundation of New Zealand, 2014.

15 Heart Foundation of New Zealand. New Zealand guidelines for rheumatic fever: diagnosis, management and secondary prevention of acute rheumatic fever and rheumatic heart disease: 2014 update, 2014.

16 Jack SJ, Williamson DA, Galloway Y, et al. Primary prevention of rheumatic fever in the 21st century: evaluation of a national programme. Int J Epidemiol 2018;47:1585-93.

17 Labtests. About Labtests Auckland, 2016. Available: http://www. labtests.co.nz/about-us/about-labtests

18 New Zealand Ministry of Health. Pharmaceutical Claims Data Mart (PHARMS) data mart - data dictionary Wellington, New Zealand, 2017. Available: https://www.health.govt.nz/system/files/documents/ publications/pharmaceutical_claims data mart pharms data dictionary_v4.2_0.pdf

19 Atkinson J, Salmond C, Crampton P. NZDep2013 index of deprivation. Wellington: Department of Public Health, University of Otago, 2014

20 Stastistics New Zealand, 2013. Available: www.stats.govt.nz

21 Carapetis JR, Beaton A, Cunningham MW, et al. Acute rheumatic fever and rheumatic heart disease. Nat Rev Dis Primers 2016;2:15084.

22 Lorenz N, Ho TK, McGregor R, et al. Serological profiling of group A Streptococcus infections in acute rheumatic fever. Clin Infect Dis 2021. doi:10.1093/cid/ciab180. [Epub ahead of print: 26 Feb 2021].

23 Baker MG, Gurney J, Oliver J, et al. Risk factors for acute rheumatic fever: literature review and protocol for a case-control study in New Zealand. Int J Environ Res Public Health 2019;16. doi:10.3390/ ijerph16224515. [Epub ahead of print: 15 Nov 2019].

24 Carapetis JR, Steer AC, Mulholland EK, et al. The global burden of group A streptococcal diseases. Lancet Infect Dis 2005;5:685-94.

25 Robson B, Harris R. Hauora: Maori standards of health 4. A study of the years 2000-2005. Wellington, New Zealand: Te Rōpū Rangahau Hauora a Eru Pōmare, 2007.

26 Raynes JM, Frost HRC, Williamson DA, et al. Serological evidence of immune priming by group A streptococci in patients with acute rheumatic fever. Front Microbiol 2016;7:1119.

27 Oliver J, Pierse N, Williamson DA, et al. Estimating the likely true changes in rheumatic fever incidence using two data sources. Epidemiol Infect 2018;146:265-75.

28 Nordet P, Lopez R, Dueñas A et al. Prevention and control of rheumatic fever and rheumatic heart disease: the Cuban experience (1986-1996-2002). Cardiovasc J Afr 2008;19:135-40. 